\title{
Investigation into plant characters affecting the competitive ability of perennial ryegrass (Lolium perenne L.)
}

\author{
T. Baan Hofman and G. C. Ennik \\ Centre for Agrobiological Research (CABO), Wageningen
}

Accepted: 2 November 1979

Key words: clones, roots, soluble carbohydrate content, vegetative phase, reproductive phase

\section{Summary}

In a number of pot and tray experiments in soil and in water culture always the same clone of the six perennial ryegrass clones tested was the most competitive both in the vegetative phase and in the reproductive phase, where another clone always showed the least competitive ability.

The most competitive clone had the relatively greatest root system, the least competitive clone the relatively smallest root system.

The soluble carbohydrate content in tillers and stubbles of the clones did not yield any evidence on competitive ability.

\section{Introduction}

Perennial ryegrass is a major component of intensively managed grassland. High yields, produced by high nitrogen applications and long growing periods, often have a deleterious effect on regrowth which may result in decreased sward density and ingress of undesired species.

Attempts are made by breeders to select for types of perennial ryegrass which are more tolerant to modern techniques and to competition under conditions with high nitrogen dressing.

In the present trials the competitive ability of six clones of perennial ryegrass was studied and an investigation was made into plant characters affecting the competitive ability.

\section{Materials and methods}

The six perennial ryegrass clones used in the experiments were selected by the 
Foundation for Plant Breeding (SVP) at Wageningen. Their code numbers are 28, $39,40,48,52$ and 368 .

Seven competition experiments were carried out, of which five on a sandy soil with $\mathrm{pH}-\mathrm{KCl}$ value, organic matter content and clay fraction of 5.6, 3.7\% and $6 \%$, respectively. Two other experiments were done in water culture (halfstrength Hoagland solution). Experiments 1 through 6 were carried out in a conditioned glasshouse. Experiment 7 was outdoors. Depending on irradiation the temperature in the glasshouse ranged from 15 to $23^{\circ} \mathrm{C}$.

In experiment 6 the clones were planted at random; in the other experiments always mixtures of two clones were used, planted chequerwise at a ratio $0.5: 0.5$, with respect to the monocultures. In the experiments 5 and 7 all possible 2 by 2 combinations of the 6 clones were included. In the other experiments only a limited number of combinations was included, which were chosen in such a way as to enable the calculation of the behaviour of those combinations not included. In experiment 6 the monocultures of the clones were not included, in the other experiments they were.

When planted the tillers were about $10 \mathrm{~cm}$ long, while roots and most of the leaves had been removed. Fertilizer and water supply were kept optimal within the limits of the experimental set up. As a measure for the competitive ability the dry matter yields of the nett plots were used or in pot experiments the yield per pot. The harvested tillers, stubbles and roots were dried at $70{ }^{\circ} \mathrm{C}$.

Experiment 1 was set up in trays and was intended to determine the competition order of the clones kept in the vegetative phase during the experiment. Plots of $18 \mathrm{~cm} \times 18 \mathrm{~cm}$ were set out in the trays.

Experiment 2 was equal to experiment 1 with the only difference that daylight was supplemented with seven $400 \mathrm{~W}$ HPL lamps per $2 \mathrm{~m}^{2}$ up to 17 hours per 24 hours.

Experiment 3 was carried out as a water culture in pots with a content of 1.3 litres. The nutrient solution was refreshed sufficiently frequent to meet the mineral requirements of the plants.

Experiment 4 was set up as experiment 3 with the difference that the same supplementary light was applied as in experiment 2 .

Experiment 5 was an experiment in trays with the objective of determining the competition order of the clones, when the reproductive phase of the plants occurred during the experimental period. Plots of 36 by $36 \mathrm{~cm}$ were set out in the trays.

Experiment 6 was also planted in trays with pregrown plants of 25 tillers each complete with roots and soil. The margin rows and the centre part of the $0.5 \mathrm{~m}^{2}$ trays were harvested separately.

The aim of experiment 7 was to obtain some insight into the size of the root systems of the six clones in monocultures as well as in mixtures. This experiment was carried out in Mitscherlich pots. Per cut two parallels were harvested completely (including roots), the yield figures are averages of two observations.

Table 1 shows a scheme of the most important data. 
Table 1. Survey of the most important experimental data.

\begin{tabular}{|c|c|c|c|c|c|c|c|c|}
\hline Exp. & Soil & $\begin{array}{l}\text { Water } \\
\text { culture }\end{array}$ & Glasshouse & Outdoors & Pots & Trays & $\begin{array}{l}\text { Suppl. } \\
\text { light }\end{array}$ & $\begin{array}{l}\text { Monoculture } \\
\text { included }\end{array}$ \\
\hline 1 & + & & + & & & + & & + \\
\hline 2 & + & & + & & & + & + & + \\
\hline 3 & & + & + & & + & & & + \\
\hline 4 & & + & + & & + & & + & + \\
\hline 5 & + & & + & & & + & & + \\
\hline 6 & + & & + & & & + & & \\
\hline 7 & + & & & + & + & & & + \\
\hline
\end{tabular}

\section{Theoretical background}

The calculation method used is based on the competition theories as described by de Wit (1960) and de Wit \& van den Bergh (1965).

The relative yield has been defined as $r=O / M$, in which $O$ is the yield of a clone in mixture and $M$ the yield of the same clone in monoculture.

In competition experiments in which the total plant density in monoculture and mixture is the same (replacement series), the expected value of the sum of the relative yields of two grass species (clones) $a$ and $b$ in a mixture is equal to 1 (de Wit, 1960; van den Bergh \& Elberse, 1970; Trenbath, 1974):

$$
r_{\mathrm{a}}+r_{\mathrm{b}}=\mathrm{RYT}=1
$$

RYT $=$ Relative Yield Total. When RYT $=1$ the clones will replace each other, the mixture will not yield higher than the highest yielding monoculture. When additional space is available to clone a or b RYT will be $>1$.

The relative replacement rate $(\varrho)$ is a measure for the rate at which one clone will replace the other (de Wit \& van den Bergh, 1965):

$$
{ }^{n l} \varrho_{a b}=\frac{{ }^{n} r_{\mathrm{a}} /{ }^{1} r_{\mathrm{a}}}{{ }^{\mathrm{n}} \boldsymbol{r}_{\mathrm{b}} /{ }^{1} \boldsymbol{r}_{\mathrm{b}}}
$$

in which ${ }^{n l} \varrho_{\mathrm{ab}}$ is the relative replacement rate of clone a with respect to clone $\mathrm{b}$ in the nth harvest with respect to the first harvest. ${ }^{1} r_{\mathrm{a}}$ and ${ }^{\mathrm{n}} \boldsymbol{r}_{\mathrm{a}}$ are the relative yields of clone $a$ in the first and in the $n$th harvest, respectively, and ${ }^{1} r_{b}$ and ${ }^{n} r_{b}$ the relative yields of clone $b$ in the first and in the nth harvest, respectively.

Another measure for the rate at which one clone is replaced by another without using the monocultures, is the relative reproductive rate $(\alpha)$ (de Wit $\&$ van den Bergh, 1965):

$$
{ }^{n l} \alpha_{\mathrm{ab}}=\frac{{ }^{\mathrm{n}} O_{\mathrm{a}} /{ }^{1} O_{\mathrm{a}}}{{ }^{\mathrm{n}} O_{\mathrm{b}} /{ }^{1} O_{\mathrm{b}}}
$$

in which ${ }^{n l} \alpha_{a b}$ is the relative reproductive rate of clone a with respect to clone $b$ 
in the nth harvest with respect to the first. ${ }^{1} O_{\mathrm{a}}$ and ${ }^{\mathrm{n}} \mathrm{O}_{\mathrm{a}}$ are the yields of clone $\mathrm{a}$ in the first and in the nth harvest of the mixture, respectively, and ${ }^{\mathrm{l}} O_{\mathrm{b}}$ and ${ }^{\mathrm{n}} O_{\mathrm{b}}$, those of clone $b$. So long as the ratio of the yields of the monocultures of $a$ and $b$ does not change in the course of the time, $\varrho$ and $\alpha$ will give the same result.

The $\varrho^{-}$or $\alpha$-values found for the different cuts are plotted on a logarithmic scale against time; by connecting the points with each other 'course lines' are found. When ${ }^{\mathrm{nl}} \varrho_{\mathrm{ab}}>1$ clone a has the advantage of clone $\mathrm{b}$ and when ${ }^{\mathrm{Il}} \varrho_{\mathrm{ab}}<1$ clone $\mathrm{b}$ gains on clone $\mathrm{a}$. This also holds for the $a$-values. According to the definitions $\varrho_{\mathrm{b} . \mathrm{a}}=\frac{1}{\varrho_{\mathrm{a} . \mathrm{b}}}$ (similarly $\left.\alpha_{\mathrm{b} . \mathrm{a}}=\frac{1}{\alpha_{\mathrm{a}, \mathrm{b}}}\right)$.

When in the experiment a few mixtures are not included, the $o$-value for the mixture b.c not included can be calculated, when the experiment does include the mixtures a.b and a.c:

$$
\varrho_{\mathrm{b} . \mathrm{c}} \text { calculated }=\frac{1}{\varrho_{\mathrm{a} . \mathrm{b}}} \times \varrho_{\mathrm{a} . \mathrm{c}}
$$

With this equation the actual $\varrho$-value of a mixture included in the experiment can be compared to $\varrho$-values calculated for the same mixture from those of other mixtures. Good correspondence between these values makes the results of this experiment more reliable. In the same way $\alpha$-values can be calculated and compared.

\section{Results}

Competition in the vegetative phase

In experiments 1 and 2 of all the clones tested clone 39 was found to be the most competitive and clone 40 the least competitive.

Contrary to experiments 5 and 7 , the monocultures in experiments 1 and 2 appeared to be not completely independent of the surrounding plots, due to the small surface of the 'plots'. Therefore in experiment 1 and 2 the relative reproductive rate $(a)$ was chosen as a measure for the competitive ability and in experiment 5 the relative replacement rate $(\varrho)$, in which the monocultures are also included. The course lines for the relative reproductive rate of clone 39 with respect to the other clones in experiments 1 and 2 have been plotted in Fig. 1. Between the course lines with and without supplementary light there is good correspondence. Clone 39 gains on all the other clones ( ${ }^{\mathrm{nl}} \alpha_{39 . \mathrm{x}}>1$ ). Clone 28 and especially 52 and 368 are replaced less rapidly by clone 39 than the clones 40 and 48.

For mixture 39.40 it was possible in addition to the directly determined course line also to calculate a course line from other mixtures included in the experiment. There is good correspondence between the actual and the calculated values.

Experiments 3 and 4 were terminated prematurely, the grass in the water culture was diseased after the second and the third cut, respectively. The $\alpha$-values found for these experiments have been plotted in Fig. 2 in a logarithmic scale 


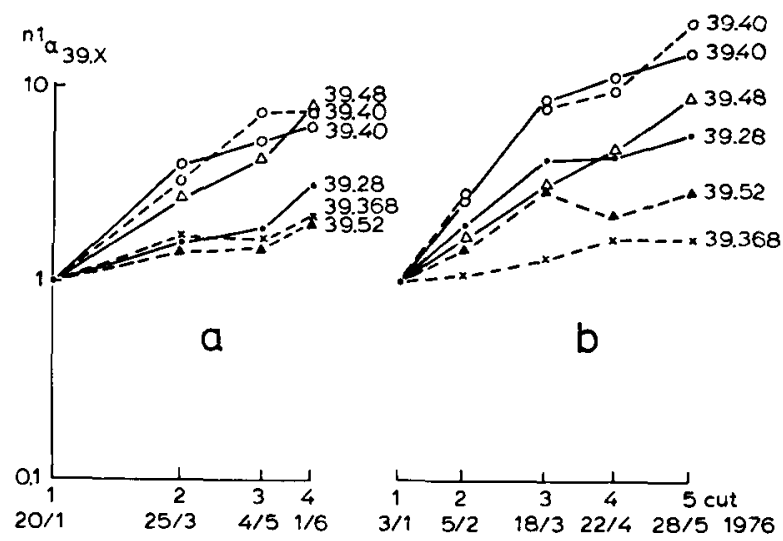

Fig. 1. Experiment 1 and 2 ( $a=$ without and $b=$ with supplementary light in 1976). 'Course lines' of the relative reproductive rate $(\alpha)$ of clone 39 with respect to the other clones in the nth harvest with respect to the 1 st harvest.

Continuous lines: mixtures occurring in the experiment; interrupted lines: values calculated. Correlation between ${ }^{n l} \alpha_{39.40}$ measured and calculated in Figs. a and $b: r=0.89$ and $r=0.94$, respectively.

per cut against the $\alpha$-values of the corresponding experiments 1 and 2 in soil. In Figs. $2 a$ and $2 b$ the points are situated near the $45^{\circ}$-line which runs through the origin, the correlation coefficient is high. This value is appreciably lower for the experiments without supplementary light (Fig. 2c), presumably the grass in the water culture was already partly diseased in the second cut. The three graphs in Fig. 2 show that also in water culture clone 39 is gaining $\left({ }^{\mathrm{nl}} \alpha_{39 . \mathrm{x}}>1\right.$ and $\left.{ }^{\mathrm{nl}} \alpha_{\mathrm{x} .39}<1\right)$.

In experiment 6 the ${ }^{\mathrm{nl}} \alpha_{39.40}$-value in the fifth cut of the margin rows and the centre rows of the trays is 5.6 and 1.9 , respectively. The belonging yield differences are very great (Table 2). Clones 40 and 48 hardly showed any regrowth

Table 2. Experiment 6. Mixture of the 6 clones. Dry matter yields in $g$ per plant per cut and total, separately for the margin rows and for the centre rows. Average of 3 parallels.

\begin{tabular}{|c|c|c|c|c|c|c|c|c|c|c|c|c|}
\hline \multirow{2}{*}{$\begin{array}{l}\text { Clone } \\
\text { harvest } \rightarrow\end{array}$} & \multicolumn{6}{|c|}{ Margin rows } & \multicolumn{6}{|c|}{ Centre rows } \\
\hline & & 2 & 3 & 4 & 5 & total & 1 & 2 & 3 & 4 & 5 & total \\
\hline 28 & 4.4 & 6.5 & 11.1 & 12.2 & 12.1 & 46 & 4.4 & 3.8 & 5.9 & 6.2 & 4.9 & 25 \\
\hline 39 & 6.2 & 10.7 & 20.2 & 20.5 & 24.6 & 82 & 7.4 & 10.2 & 15.6 & 11.7 & 12.2 & 57 \\
\hline 40 & 4.9 & 4.6 & 5.8 & 3.6 & 3.5 & 23 & 4.2 & 3.0 & 4.1 & 4.2 & 3.7 & 19 \\
\hline 48 & 4.9 & 5.0 & 7.1 & 5.6 & 4.5 & 27 & 4.2 & 3.2 & 3.8 & 3.4 & 2.6 & 17 \\
\hline 52 & 4.9 & 7.1 & 10.2 & 8.2 & 8.1 & 39 & 5.1 & 6.5 & 8.5 & 6.6 & 4.7 & 31 \\
\hline 368 & 6.4 & 6.4 & 10.6 & 12.6 & 14.1 & 50 & 4.9 & 4.4 & 6.0 & 7.0 & 6.1 & 29 \\
\hline
\end{tabular}



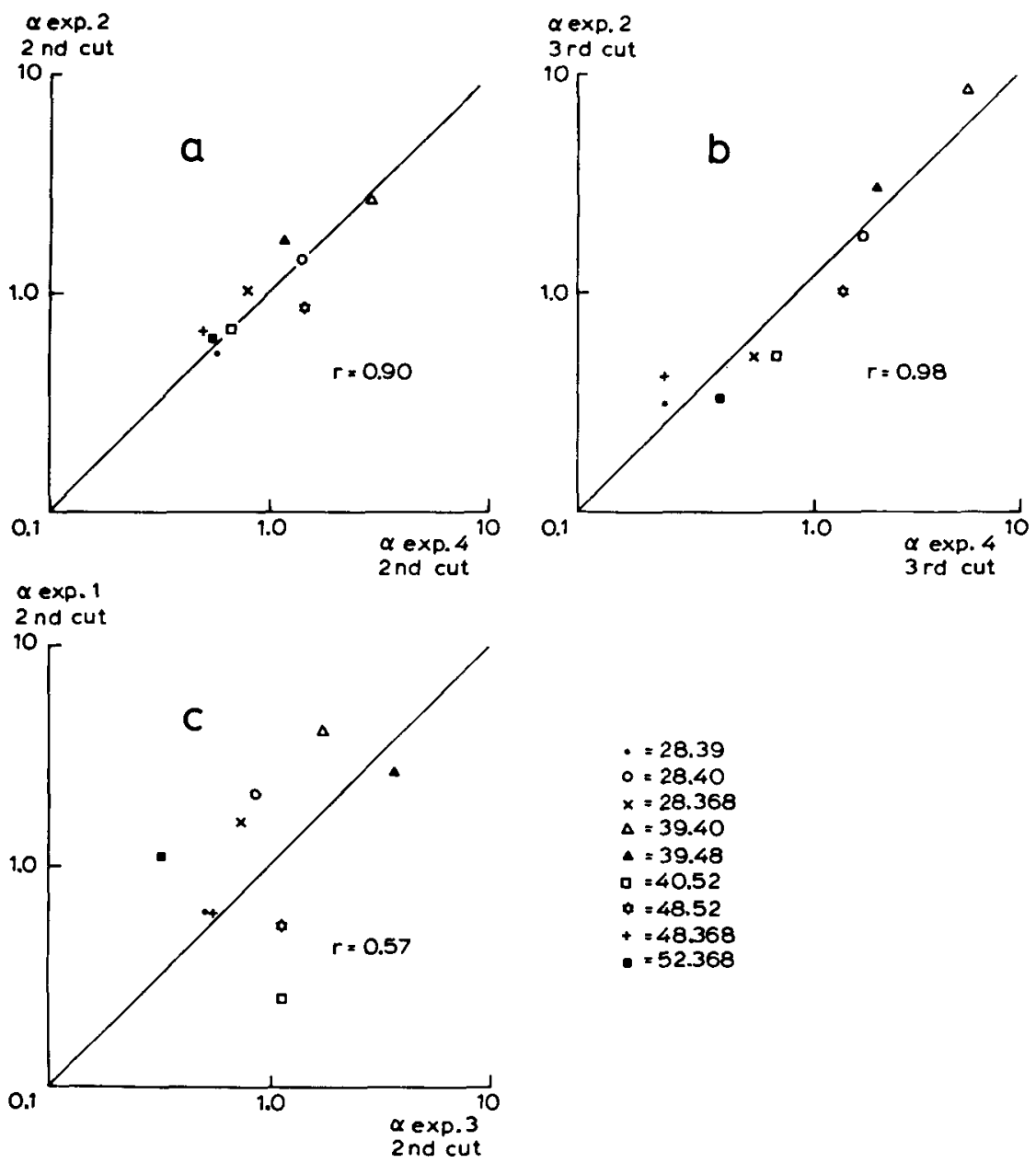

Fig. 2. Experiments 1,2,3 and 4. a-values for the mixtures in soil, plotted per cut against those for the mixtures in mineral solution. $a=$ with supplementary light, 2 nd cut; $b=$ item, for 3rd cut; $\mathrm{c}=$ without supplementary light, 2nd cut.

after the 5th cut; the plants were dying. The highly competitive clones 39 and 368 are more favoured by additional light than the clones that are rapidly replaced (compare margin rows with respect to the centre rows in Table 2 and experiments with supplementary light (Fig. 1b) with respect to no supplementary light (Fig. 1a)).

The ${ }^{\mathrm{nl}} \varrho_{39 . \mathrm{x}}$-values (relative replacement rates) in exp. 7 are generally greater than unity (Table 3), clone 39 is the most competitive.

The correlation between the actual and calculated $\varrho$-values for the tillers in exp. 7 is considerably lower $(r=0.65)$ than that for the $\vartheta^{-}$and $\alpha$-values in experiments 5, 1 and 2 (Figs. 4 and 1 ). 
Table 3. Experiment 7. The relative replacement rate at which clone 39 replaces the other clones in the nth harvest with respect to the 1st harvest ( $\mathrm{nl}_{\left.\varrho_{39 . x}\right)}$ ) for the dry matter yield of the tillers.

\begin{tabular}{llll}
\hline Mixture & Harvest & & \\
\cline { 2 - 4 } & 2 & 3 & 4 \\
39.28 & 1.96 & 2.38 & 2.70 \\
39.40 & 0.99 & 1.86 & 2.45 \\
39.48 & 0.80 & 1.94 & 2.15 \\
39.52 & 0.85 & 1.54 & 1.21 \\
39.368 & 0.99 & 0.96 & 1.46 \\
\hline
\end{tabular}

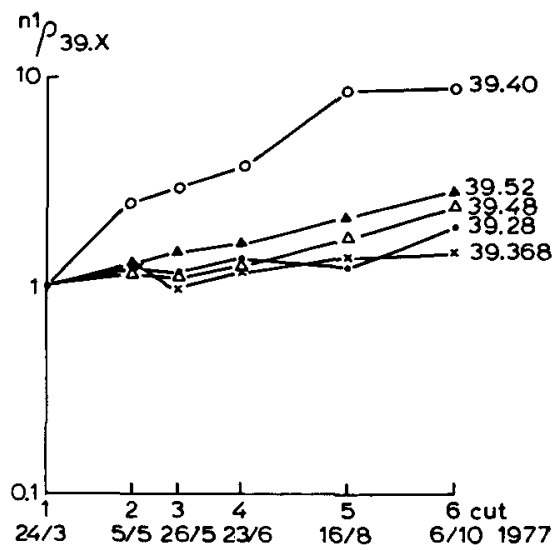

Fig. 3. Experiment 5 in 1977. 'Course lines' for the relative replacement rate $(\varrho)$ of clone 39 with respect to the other clones in the nth cut with respect to the 1 st cut.

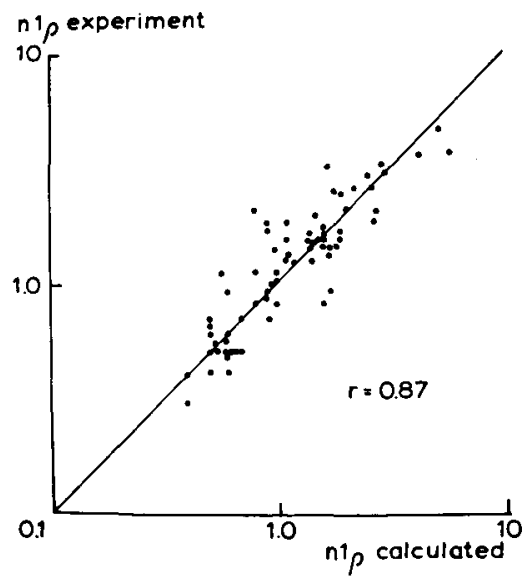

Fig. 4. Experiment 5. Relation between the actual nl $\varrho$-values and those calculated for the 2nd through 6th cuts. 
This is partly due to a deviation of clone 52 in this experiment. RYT (section 3 ) of the 39.52 mixture is $>1$, which may indicate that one of the clones had extra space available. For explanation see the discussion.

\section{Competition in the reproductive phase}

In experiment 5 planted in November 1975, in the first cut on 24 March 1976 all the clones had reproductive tillers. In the second cut on 5 May clone 52 showed most reproductive tillers, in the other clones they occurred infrequently. It is not known which percentage of the planted and newly formed tillers became reproductive. After the second cut the clones can be considered as vegetative.

The $\varrho_{39 . x}$-values in experiment 5 are higher than 1 (Fig. 3). Clone 40 as in the experiments with vegetative clones, here too is replaced most rapidly. According to results not mentioned in this paper, the second most competitive clone, 368 , replaces the other clones in almost the same order as did clone 39.

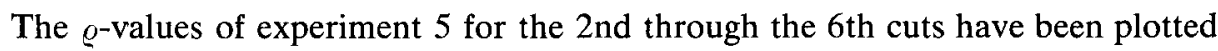
in Fig. 4 against the calculated $\varrho$-values (section 3 ). The high correlation coefficient shows that there is good correspondence between the actual and the calculated values.

\section{Distribution of dry matter by clones in monoculture}

With regard to the tiller yield (= overground mass minus stubbles) at the first harvest of experiment 7 (done outdoors) the clones can be divided into two groups differing in dry matter yield. The clones 48, 52 en 368 are early and rapidly growing clones in contrast to clones 28,39 and 40 which yield appreciably lower at the first cut (Fig. 5b). In the second cut clone 28 is still a low yielder; 40 and 39 are then the highest yielding clones, but in the third cut they are lower again.

The stubble mass of all the clones increases gradually with time (Fig. 5c). Clones 40 and 48 , the least competitive in the mixtures, leave more stubble after harvesting than the competitive clone 39 does.

The root mass (dry matter) of clone 39 is largest, that of clone 40 smallest, the other clones are intermediary (Fig. 5d). After the second harvest the root masses of the clones hardly increase, except for clone 39 of which the root mass continued to increase linearly until the 4 th harvest.

The total dry matter yield varies between clones, especially in the first and second cut (Fig. 5a), which is mainly caused by a difference in shoot yield. Among the group of 6 clones, clone 28 is striking because of the very gradual increase in total dry matter yield. The advantage of clone 39 at the 4th harvest is only small and due to the continued growth of the root system.

The difference in dry matter yield of the shoots between clones 39 and 40 is small (Fig. 5b), the size of the root systems determines for a large extent the difference in shoot/root ratio (Fig. 6). Clone 40 shows a high tiller yield in the 2nd cut (Fig. 5b), the root system does not increase after the first cut, and so the shoot/root ratio at the 2 nd cut is high. The (shoot + stubble)/root ratio closely corresponds with the shoot/root ratio. 


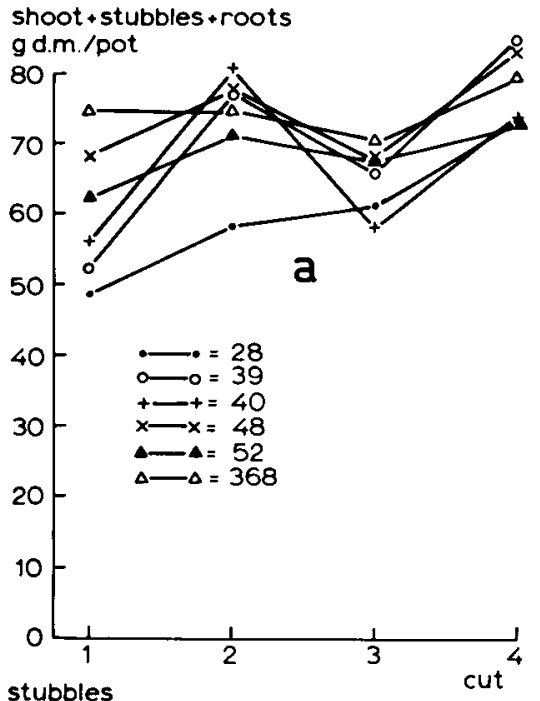

stubbles

g d.m. / pot

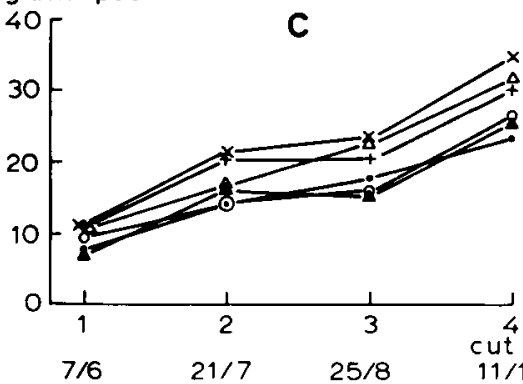

C

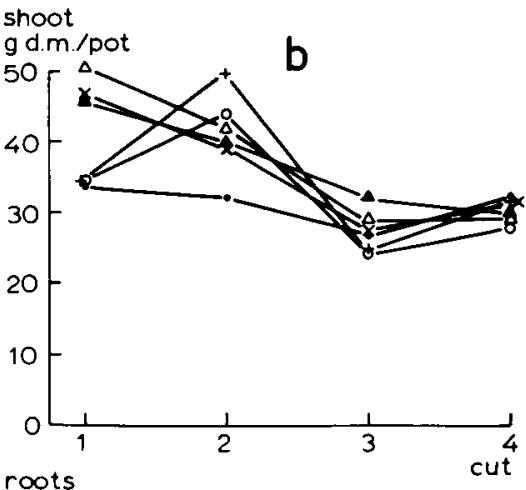

roots

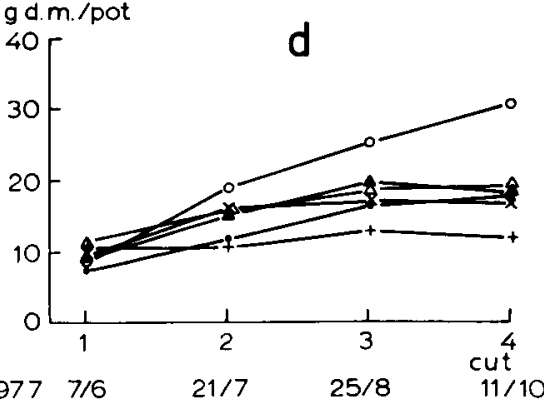

Fig. 5. Experiment 7 in 1977. Dry matter yields of the clones in monoculture during the experimental period from April to October 1977. a, shoot + stubbles + roots; b, shoot; c, stubbles; d, roots.

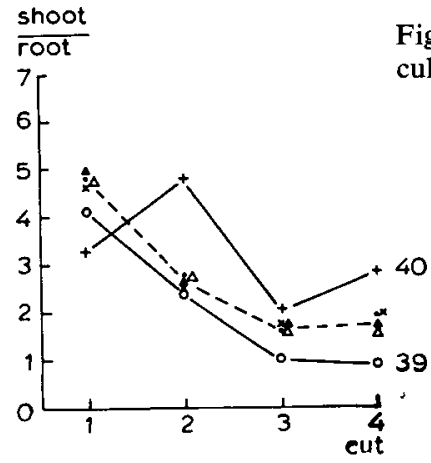

Neth. J. agric. Sci. 28 (1980) 


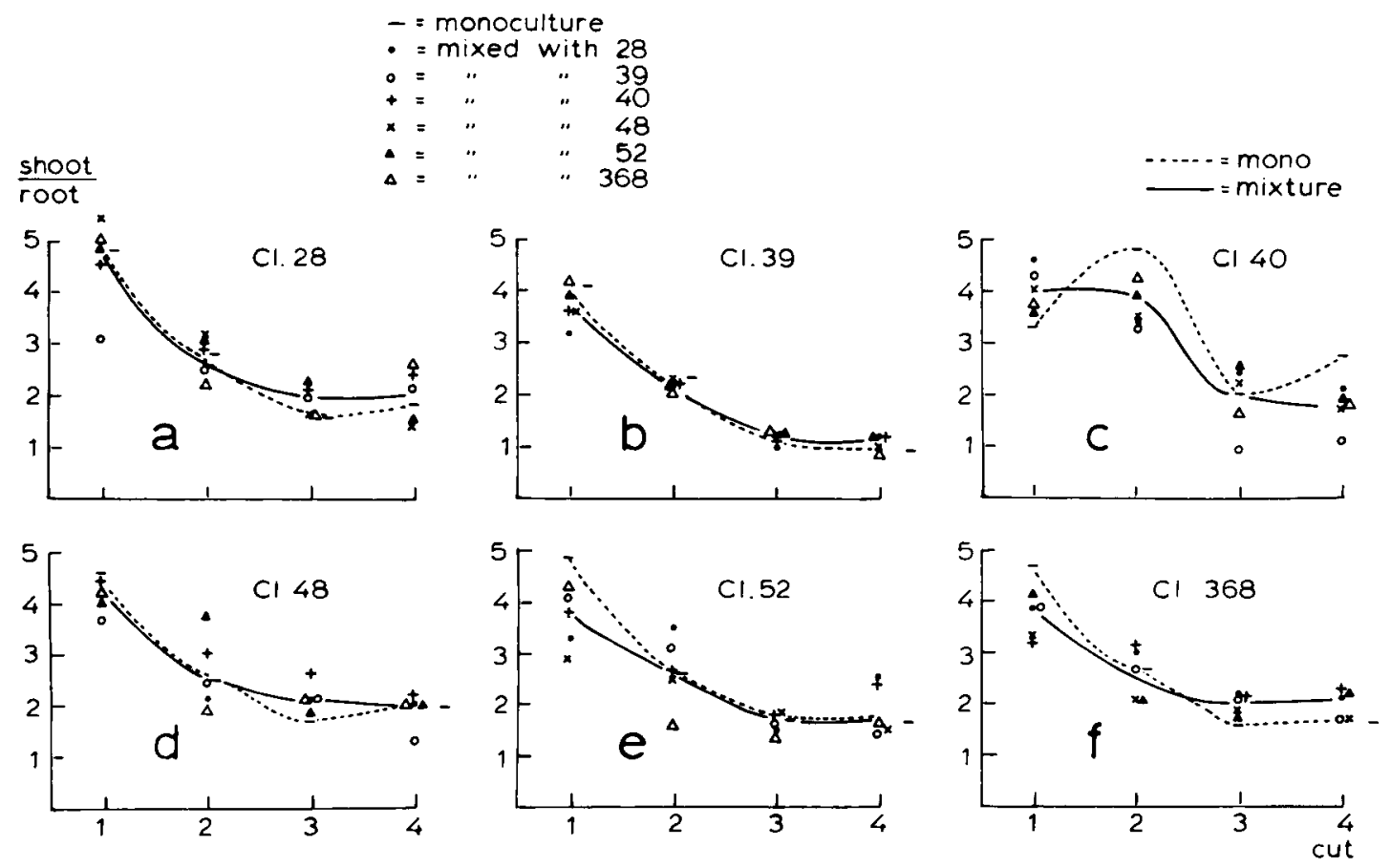

Fig. 7. Experiment 7. Shoot/root ratio of each clone competing with the other 5 clones (continuous line) compared with the shoot/root ratios of the clones in monoculture (interrupted line). An explanation for the deviating points can be read in the text.

Distribution of dry matter by clones in mixture

Apart from a few deviations the shoot/root ratios of the clones in mixture (experiment 7) correspond well with those of the monocultures (Fig. 7). In the mixture 28.39, first harvest, the soil in one of the pots was wet, due to a blocked outlet, in Figs. $7 \mathrm{a}$ and $7 \mathrm{~b}$ this is demonstrated by a decreased shoot/root ratio. Clone 40 in competition with clone 39 (the most competitive clone) at the 3 rd and 4th harvests did not regain the same shoot/root ratio as in the mixtures with the other clones (Fig. 7c). Contrary to the normal situation under conditions of optimum N supply (Ennik 1966), the plants of clone 40 were unable to restore their former shoot/root ratio. This also applies to clone 48 in the mixture 48.39 4th harvest (Fig. 7d).

The high tiller yield of clone 40 in the 2 nd harvest is also reflected in the shoot/root ratio in the mixtures, however, to a less extent than in the monoculture because of tiller competition (Fig. 7c).

The points for the shoot/root ratios are rather scattered, except for those of clone 39. This clone is apparently highly dominant, the others are more easily affected. Presumably this will not always be the same in all cases and many factors will affect this. The scatter of clone 39 is also smaller, because at equal shoot yields of the clones the greater root mass of clone 39 will bring about a smaller scatter of the shoot/root quotient for mathematical reasons. 


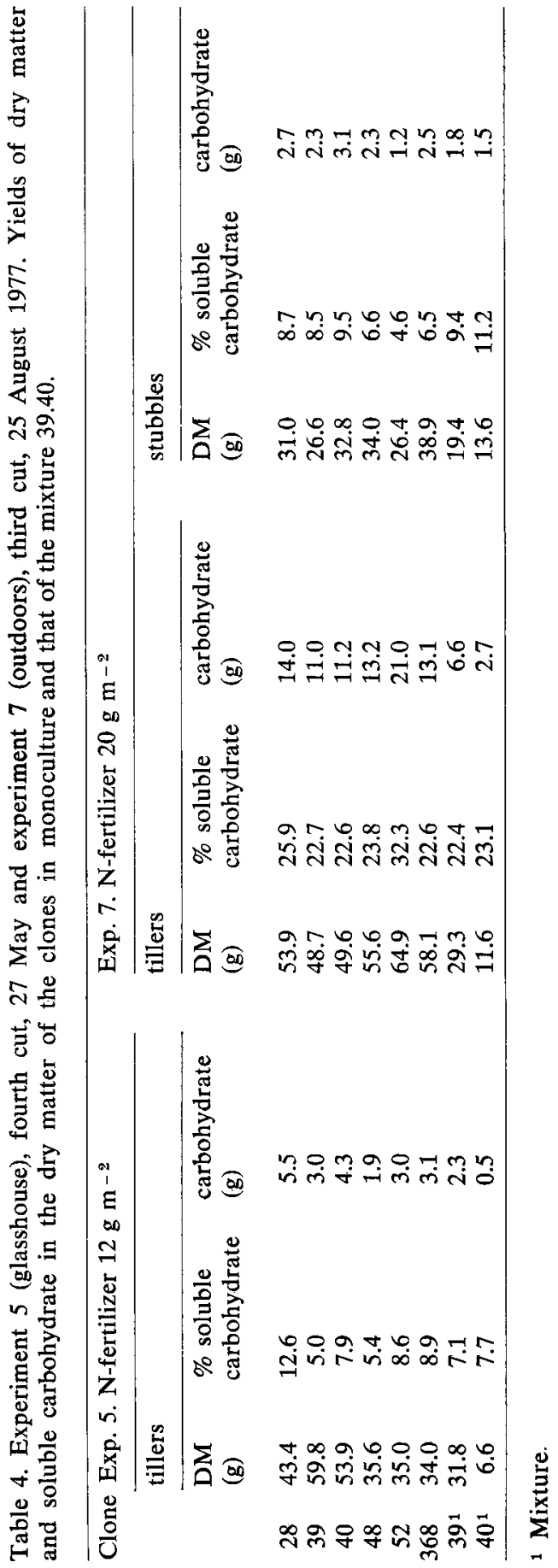

Neth. J. agric. Sci. $28(1980)$ 


\section{Soluble carbohydrates}

Replacement of the other clones by clone 39 takes place at a lower rate in experiment 7 than in experiment 5 (lower $\varrho_{39 . x}$-values). In experiment 7 the proportion of light to temperature is more favourable to perennial ryegrass than in the glasshouse experiments. Among others, this is apparent from the lower contents of soluble carbohydrates in the tillers of the glasshouse experiment 5 compared to those in experiment 7 outdoors (Table 4). The plants were not grown in the same month and day length in August is somewhat shorter than in May. Comparison is therefore not quite correct, but the difference is striking.

Clone 39 in monoculture does not strikingly differ in percentage and weight of soluble carbohydrates within one experiment compared with other clones. In the mixture 39.40 the content of soluble carbohydrates of clone 40 , as in the monocultures, is equal to or higher than that of clone 39 (Table 4). Deficiency of soluble carbohydrates therefore could not have been the reason for clone 40 to be retarded in the mixture.

When the stubble in experiment 7 was harvested it was impossible to separate the living, dying or dead material. The content of soluble carbohydrates in the stubble is therefore considerably lower than in the tillers (Table 4). None of the six clones has a percentage or weight of soluble carbohydrates deviating to such an extent that it could be used in explanation of the difference in competitive ability.

\section{Discussion}

In all the experiments (both in soil and in nutrient solution, and with clones in the vegetative and in the reproductive phase) clone 39 is the most competitive and clone 40 the least competitive.

The most striking difference between the clones 39 and 40 is the root system (Fig. 5d). That a relatively small root system should have been limiting in the uptake of minerals and water does not seem likely. In experiment 7 e.g. an equally high dressing was applied before each cut. The clones produced high dry matter yields in the 1 st and 2 nd cuts, but those in the 3 rd and 4 th cuts were lower (Fig. 5b). In the experiments in water culture the supply of minerals was always sufficient and continued, clone 39 was the most competitive in these experiments as well and clone 40 at the greatest disadvantage (Fig. 2).

Clone 40 with its small root system was able to produce high yields, as in demonstrated by Fig. 5b, second cut. The negative correlation between the yields of successive cuts, as occurring in clone 40 , has been observed frequently in grass and is not due to insufficient mineral supply.

The content of soluble carbohydrates in the tillers of experiment 7, 3rd cut (Table 4) is so high that root growth of all the clones, similarly as with nitrogen shortage in the plant, might be stimulated (Brouwer, 1963). Only the root mass of clone 39 increased. If clone 39 had taken up more nitrogen than the other clones, it might have been expected that the root system of clone 39 might have grown to a less extent than those of the other clones. This is not the case. The 
low shoot/root ratio of clone 39 is therefore genetically established.

Cocksfoot in competition with perennial ryegrass is less rapidly replaced as the cutting frequency increases. Van den Bergh (1968) suggested that the green stubble left on the field by cocksfoot can develop more photosynthetic activity than the brown stubble of perennial ryegrass, thus providing more carbohydrates for regrowth. However, the stubble of clone 39 is no greener than those of 48 and 368 , therefore, clone 39 in this respect is not in advantage of the other clones. Clones 39, 48 and 368 are the most prostrate of the six; 48, 368 and 40 leave most stubbles (Fig. 5c), while clones 40 and 48 show little competitive ability. Stubble mass therefore neither explains the competitive ability of clone 39 .

Van den Bergh and Elberse (1970) suggested that at high yields the species with the longest tillers would be favoured in competition compared to the somewhat more prostrate species. Clone 52 has longer and more erect growing tillers than the other clones. In the Mitscherlich pots these longer tillers probably inclined further, thereby intercepting more light. The higher percentage of soluble carbohydrates in the tillers of this clone in experiment 7 (Table 4) confirms this. Rhodes (1968) did not find RYT $>1$ in mixtures of prostrate and erect growing ryegrass types. In experiment 5 (data not included in this paper) RYT $>1$ was neither found in the mixture 39.52. In this experiment the plants did not grow in pots, but in plots with margin rows and consequently the plants could not incline. Clone 39 does not have the advantage of long tillers, but it is successful in the competition with other clones.

On the basis of the results of this series of experiments we draw the conclusion that the order in the size of the root systems of the clones is equal to that in competitive ability. Whether this relation is accidental or causal will have to be demonstrated by further experiments.

\section{Acknowledgement}

We are indebted to miss A. H. van Rossem for translating the manuscript.

\section{References}

Bergh, J. P. van den, 1968. An analysis of yields of grasses in mixed and pure stands. Versl. landbouwk. Onderzoek. 714 (Agric. Res. Rep. 714), 71 pp.

Bergh, J. P. van den \& W. Th. Elberse, 1970. Yields of monocultures and mixtures of two grass species differing in growth habit. J. appl. Ecol. 7: 311-320.

Brouwer, R., 1963. Some aspects of the equilibrium between overground and underground plant parts. Jaarb. IBS 1963: 31-39.

Ennik, G. C., 1966. Influence of clipping and soil fumigation on shoot and root production of perennial ryegrass and white clover. Jaarb. IBS 1966: 11-18.

Rhodes, I., 1968. Advance note on research: Yield of contrasting ryegrass varieties in monoculture and mixed culture. J. Br. Grassl. Soc. 23: 156-158.

Trenbath, B. R., 1974. Biomass productivity of mixtures. Adv. Agron. 26: 177-210.

Wit, C. T. de, 1960. On competition. Versl. landbouwk. Onderzoek. 66.8, 82 pp.

Wit, C. T. de \& J. P. van den Bergh, 1965. Competition between herbage plants. Neth. J. agric. Sci. 13: 212-221.

Neth. J. agric. Sci. $28(1980)$ 